At week 10, CD40 expression on ductal epithelial and endothelial cells in NOD mice of vaccine group was significantly decreased positive staining. CD40 mRNA expression level showed a significantly reduction compared to vector group $(0.51$ \pm 0.31 vs. $1.56 \pm 0.53, p<0.05$ )

3) Down-regulation of lymphocytes infiltration in the salivary glands of mice in vaccine group

At week 10, infiltration of lymphocytes was inhibited in treated group while increased in control group $(F=5.275, P<0.05)$. FS was significantly decreased in vaccine group as compared to NS group $(2.00 \pm 1.73$ vs. $11.33 \pm 5.51, \mathrm{P}<0.05)$. Average weight of wet salivary gland and the ratio of average salivary gland weight to body weight of NOD mice in vaccine group were significantly lower than that in control groups ( $p<0.05$ and $p<0.05$, respectively).

4) CD40 DNA vaccine reduced the expression of TNF- $\alpha$ and IL-6 in the salivary glands

In vaccine group, the expression level of TNF- $\alpha$ mRNA in salivary glands were declined significantly as compared to baseline $(0.41 \pm 0.25$ vs. $0.98 \pm 0.16, p<0.05)$ and IL-6 mRNA expression was down-regulated compared with control groups at week 10 ( $p<0.05 ; p<0.01$, respectively).

5) Disturbances in spleen DC and plasma cell subpopulations

At week 6 , the total numbers of $C D 11 c^{+} D C$ decreased as compared with two control groups $\left(p<0.05\right.$ and $p<0.05$, respectively). CD11c ${ }^{+} D C$ and CD $19^{+}$CD $138^{+}$plasma cells were significantly reduced compared to basal level $(\mathrm{p}<0.01$ and $\mathrm{p}<0.05$, respectively).

6) Level of ANA reduced in the vaccine group

At week 10, the expression of ANA in HEp-2 cells is strong positive (++++) in both control groups, but only positive (+) in vaccine group.

Conclusions: These findings indicate that CD40 DNA vaccine can downregulate the expression of proinflammatory cytokines of TNF- $\alpha$ and IL- 6 , decrease the percentage of DCs and plasma cells, ameliorate the pathologic change in NOD mice with SS, and thus inhibit the autoimmune inflammation.

Disclosure of Interest: None declared

DOI: 10.1136/annrheumdis-2018-eular.3939

\section{FRI0256 EFFECTS OF ANIFROLUMAB ON OXIDATIVE STRESS AND MACROPHAGE ACTIVATION: NOVEL BIOMARKERS AND IMPACT OF TYPE I INTERFERON BLOCKADE IN SYSTEMIC LUPUS ERYTHEMATOSUS}

$\underline{X}^{\text {X. Guo }}{ }^{1}$, S. Wang ${ }^{1}$, L. Wang ${ }^{2}$, L.K. Roskos ${ }^{1}$, W.I. White ${ }^{1} .{ }^{1}$ Medimmune;

${ }^{2}$ AstraZeneca, Gaithersburg, USA

Background: Type I interferons (IFNs) drive several aspects of systemic lupus erythematosus (SLE) etiology and pathophysiology. Anifrolumab, a fully human anti-IFN- $\alpha$ receptor monoclonal antibody, substantially reduced disease activity compared with placebo in a Phase Ilb study of SLE patients. ${ }^{1}$ Previous results indicated beneficial effects of anifrolumab on dysregulated lymphocyte, neutrophil, and complement systems in SLE. ${ }^{2}$ However, the impact of type I IFN blockade remains to be fully elucidated.

Objectives: We characterised biomarkers for oxidative stress and macrophage activation in SLE and assessed downstream effects of anifrolumab on those two key disease pathways.

Methods: In the MUSE study, ${ }^{1} 305$ patients with moderate to severe SLE were randomised in a 1:1:1 ratio to receive placebo or anifrolumab (300 or 1,000 mg) every 4 weeks for 48 weeks. A four-gene expression assay was used to define type I IFN gene signature (IFNGS) test status. In our study, four protein biomarkers were measured in sera of healthy controls (HCs) and SLE patients with samples available at baseline, and 85, 169, and 365 days after administration, using a multiplex luminex immunoassay. Wilcoxon rank-sum test was used to evaluate baseline protein concentrations and posttreatment changes between different groups.

Results: Two oxidative stress biomarkers, peroxiredoxin 4 (PRDX4) and aldose reductase (AKR1B1), were found in greater serum concentrations in 195 SLE patients than in $20 \mathrm{HCs}(p<0.01)$. Their increased concentrations were associated with greater SLEDAI scores and abnormal anti-dsDNA concentrations $(p<0.05)$. PRDX4 concentrations were greater for IFNGS test-high $(n=144)$ vs IFNGS testlow $(n=51)$ patients $(p<0.01)$, whereas AKR1B1 was not associated with type I IFNGS-test status. Anifrolumab suppressed PRDX4 in IFNGS test-high patients compared with placebo $(\mathrm{p}<0.05)$. In contrast, no significant changes were observed for AKR1R1 after anifrolumab treatment. Folate receptor 3 (FOLR3) and CD163 are markers of activated macrophages, the former of which has not been characterised in SLE. Our results demonstrated greater concentrations of both FOLR3 and CD163 in IFNGS test-high than in IFNGS test-low patients and HCs, along with significant associations with SLEDAI score and anti-dsDNA concentrations. Anifrolumab reduced serum concentrations of both FOLR3 and CD163 in IFNGS test-high but not IFNGS test-low patients vs. placebo $(p<0.05)$. Moreover, cellular enumeration data demonstrated anifrolumab-induced upregulation of monocyte count in IFNGS test-high patients, which may result from reduced activation, apoptosis, and/or tissue infiltration of monocytes/macrophages.

Conclusions: Our results identified PRDX4 as an oxidative stress marker and FOLR3 as a macrophage activation marker associated with disease activities and type I IFNGS test status in SLE. Anifrolumab administration elicits reduced oxidative stress and decreased monocyte/macrophage activation, which may contribute to the clinical efficacy of anifrolumab in SLE patients.

\section{REFERENCES:}

[1] Furie R, et al. Arthritis Rheumatol. 2017;69:376-86.

[2] Guo X, et al. Ann. Rheum. Dis. 2016;75:118.

Disclosure of Interest: X. Guo Shareholder of: AstraZeneca, Employee of: MedImmune, S. Wang Shareholder of: AstraZeneca, Employee of: Medlmmune, L. Wang Shareholder of: AstraZeneca, Employee of: AstraZeneca, L. Roskos Shareholder of: AstraZeneca, Employee of: AstraZeneca, W. White Shareholde of: AstraZeneca, Employee of: Medlmmune DOI: 10.1136/annrheumdis-2018-eular.3842

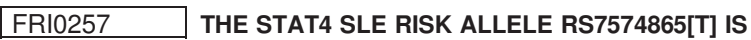 ASSOCIATED WITH INCREASED IL-12-INDUCED IFN- GAMMA PRODUCTION IN T CELLS FROM SLE PATIENTS}

N. Hagberg ${ }^{1}$, M. Joelsson ${ }^{1}$, D. Leonard ${ }^{1}$, S. Reid ${ }^{1}$, M.-L. Eloranta ${ }^{1}$, J. Mo $^{2}$, M. Nilsson ${ }^{2}$, A.-C. Syvänen ${ }^{3}$, Y.T. Bryceson ${ }^{4,5}$, L. Rönnblom ${ }^{1} .{ }^{1}$ Department of Medical Sciences, Rheumatology and Science for Life Laboratories, Uppsala University, Uppsala; ${ }^{2}$ Respiratory, Inflammation and Autoimmunity, IMED Biotech Unit, AstraZeneca, Gothenburg; ${ }^{3}$ Department of Medical Sciences, Molecular Medicine and Science for Life Laboratories, Uppsala University, Uppsala; ${ }^{4}$ Department of Medicine, Center for Hematology and Regenerative Medicine, Karolinska Institutet, Karolinska University Hospital Huddinge, Stockholm, Sweden; ${ }^{5}$ Department of Clinical Sciences, Broegelmann Research Laboratory, University of Bergen, Bergen, Norway

Background: Genetic variants in the transcription factor STAT4 are associated with increased susceptibility to systemic lupus erythematosus (SLE) and a more severe disease phenotype.

Objectives: This study aimed to clarify how the SLE-associated intronic STAT4 risk variant rs7574865[T] affects the function of immune cells in SLE.

Methods: Peripheral blood mononuclear cells (PBMCs) were isolated from 52 SLE patients in remission (SLEDAI-2K $\leq 4$ ). STAT4 and STAT1 protein levels and phosphorylation status in response to interferon (IFN)- $\alpha$, IFN- $\gamma$, or interleukin (IL) -12 were determined, before and after pre-activation of cells with phytohaemagglutinin (PHA) and IL-2, in CD56 ${ }^{\text {dim }}$ NK cells, CD56 $6^{\text {bright }}$ NK cells, B cells, $\mathrm{CD}^{+} \mathrm{T}$ cells, $\mathrm{CD}^{+} \mathrm{T}$ cells and monocytes by flow cytometry. The frequency of IFN- $\gamma^{+}$cells upon IL-12 or PMA (phorbol 12-myristate-13-acetate) stimulation and the frequency of $\mathrm{T}^{-\mathrm{bet}^{+}}$cells was determined in PHA/IL-2 pre-activated cells. Cellular responses and phenotypes were correlated with STAT4 risk allele carriership (rs7574865[T], 21 homozygous protective, 22 heterozygous and 9 homozygous risk) using an additive linear regression model. Janus kinase inhibitors (JAKi) selective for TYK2 (TYK2i, Compound 35) or JAK2 (JAK2i, BMS-911543) were evaluated for inhibition of IL-12 or IFN- $\gamma$-induced activation of SLE PBMCs.

Results: In resting PBMCs, the STAT4 risk allele was neither associated with protein levels of STAT4 or STAT1, nor cytokine-induced phosphorylation of STAT4 (pSTAT4) or STAT1 (pSTAT1). However, following PHA/IL-2-activation, $\mathrm{CD}^{+} \mathrm{T}$ cells from STAT4 risk allele carriers displayed increased levels of STAT4 $(p=0.04)$, resulting in increased pSTAT4 in response to IL-12 $(p=0.003)$ and IFN- $\alpha$ $(p=0.04)$. Analysis of $T$ cell subsets revealed that the effect was seen in $\mathrm{CD}^{2} 5 \mathrm{RA}^{+} \mathrm{CD}^{-} 7^{-}$naïve and $\mathrm{CD} 45 \mathrm{RA}^{-} \mathrm{CD} 57^{-}$memory $\mathrm{CD} 8^{+} \mathrm{T}$ cells, but not in $\mathrm{CD} 45 \mathrm{RA}^{-} \mathrm{CD} 7^{+}$memory or $\mathrm{CD}^{+} 5 \mathrm{RA}^{+} \mathrm{CD} 57^{+}$effector $\mathrm{CD} 8^{+} \mathrm{T}$ cells. A slight increase in STAT4 protein levels and IL-12-induced pSTAT4 was also observed in $\mathrm{CD}^{+}$T cells from STAT4 risk allele carriers ( $\mathrm{p}=0.08$ and $\mathrm{p}=0.09$, respectively). STAT4 risk allele carriers displayed an augmented IL-12-induced IFN- $\gamma$ production in $\mathrm{CD}^{+}$and $\mathrm{CD} 4^{+} \mathrm{T}$ cells ( $\mathrm{p}=0.03$ for both), whereas PMA-induced IL-12 production was normal $(p=0.31$ and $p=0.10$, respectively). T-bet expression was not correlated to the STAT4 genotype. The TYK2i and the JAK2i efficiently blocked $\mathrm{IL}-12$ and IFN- $\gamma$-induced activation of PBMCs from STAT4 risk patients, respectively.

Conclusions: T cells from SLE patients carrying the STAT4 risk allele rs7574865 [T] display an augmented response to IL-12 and IFN- $\alpha$. This subset of patients may benefit from JAKi treatment.

Disclosure of Interest: N. Hagberg: None declared, M. Joelsson: None declared, D. Leonard: None declared, S. Reid: None declared, M.-L. Eloranta: None declared, J. Mo Employee of: AstraZeneca, M. Nilsson Employee of: AstraZeneca, A.-C. Syvänen: None declared, Y. Bryceson: None declared, L. Rönnblom: None declared 

LUPUS (PIL)

\section{FRI0258 \\ COMPARISON OF ELISA AND MULTIPLEX TECHNIQUES FOR QUANTIFYING A URINE BIOMARKERS PANEL FOR LUPUS NEPHRITIS IN CHILDREN}

E.M. Smith ${ }^{1,2}$, M.W. Beresford ${ }^{1,2} .{ }^{1}$ Paediatric Rheumatology, Alder Hey Children's NHS Foundation Trust, ${ }^{2}$ Women and Children's Health, Institute of Translational Medicine, University of Liverpool, Liverpool, UK

Background: A urine 'biomarker panel' comprising alpha-1-acid-glycoprotein (AGP), ceruloplasmin (CP), transferrin (TF) and lipocalin-like-prostaglandin-D synthase (LPGDS) has been shown to cross-sectionally perform to an 'excellent' level for Lupus Nephritis (LN) identification in children.' Quantification of all four biomarkers by enzyme linked immunoabsorbant assay (ELISA) techniques is time consuming and costly. Therefore, novel methods of biomarker panel quantification are required to facilitate future urine biomarker led monitoring studies.

Objectives: The main objective was to compare the ability of ELISA and multiplex biomarker quantification techniques to differentiate active versus inactive LN when the biomarkers are considered individually and in combination.

Methods: The urinary biomarkers were quantified by both ELISA and a newly developed, custom multiplex platform in participants of the UK Juvenile Systemic Lupus Erythematosus (JSLE) Cohort Study. Multiplex assay development involved identification of appropriate antibody pairs, assessment of JSLE urine sample matrix effects and range finding in JSLE patient samples. Patients were categorised as having active $L N$ (renal domain BILAG score of $A, B$ and previous histological confirmation of LN) or inactive LN (renal BILAG score D or E). Firth's penalised binary regression with AUC ROC analysis was used to compare the ability of multiplex and ELISA assays to detect active LN disease state univariately and in combination.

Results: Biomarker analysis was undertaken on 54 JSLE patients (13 active, 41 in-active). Assessment of each biomarker univariately demonstrated similar AUC values regardless of the biomarker quantification technique; LPGDS (ELISA AUC 0.826, multiplex AUC 0.829), TF (ELISA AUC 0.829, multiplex AUC 0.996), CP (ELISA AUC 0.901, multiplex AUC 0.983), AGP (ELISA AUC 0.934, multiplex AUC 0.979) (see table 1). Combining the multiplex biomarker data in the same order as the original ELISA based study led to a similar progressive increase in AUC as biomarkers were added to the model (optimal model including AGP $+\mathrm{CP}$ + LPGDS+TF ELISA AUC $=0.951$, multiplex $=0.995$ ). For all biomarker combinations, the multiplex-derived AUC was higher than the ELISA AUC.

Abstract FRI0258 - Table 1. AUC values generated looking at each biomarker univariately

\begin{tabular}{lcc}
\hline Individual biomarkers & ELISA AUC & Multiplex AUC \\
\hline LPGDS & 0.826 & 0.829 \\
TF & 0.829 & 0.996 \\
CP & 0.901 & 0.983 \\
AGP & 0.934 & 0.979 \\
\hline
\end{tabular}

Abstract FRI0258 - Table 2. Comparison of biomarker combination AUC ROC values

\begin{tabular}{lcc}
\hline Biomarker combinations & ELISA AUC & Multiplex AUC \\
\hline AGP & 0.880 & 0.979 \\
AGP+CP & 0.937 & 0.986 \\
AGP+CP+LGPDS & 0.942 & 0.985 \\
AGP+CP+ LGPDS+TF & 0.951 & 0.995
\end{tabular}

Conclusions: This new LN urine biomarker panel multiplex assay has been shown to display a comparable ability for active $\mathrm{LN}$ disease state identification as compared to existing ELISA techniques. The major advantage to this approach is that it reduces cost, processing time and the volume of sample required, as compared to ELISA techniques, representing a key enabler for future clinical studies.

\section{REFERENCE:}

[1] Smith EM, Jorgensen AL, Midgley A, Oni L, Goilav B, Putterman C, Wahezi D, Rubinstein T, Ekdawy D, Corkhill R, et al. International validation of a urinary biomarker panel for identification of active lupus nephritis in children. Pediatr Nephrol 2017:32:283-295.

Disclosure of Interest: None declared DOI: 10.1136/annrheumdis-2018-eular.5187
B. Jacobs ${ }^{1}$, H. Leiss ${ }^{2}$, I. Gessl ${ }^{2}$, A. Puchner ${ }^{2}$, J. Smolen ${ }^{2}$, G. Stummvoll ${ }^{2}$. ${ }^{1}$ Department of Internal Medicine, Providence Saint Vincent Medical Center, Portland, USA; ${ }^{2}$ Department of Rheumatology, Medical University of Vienna, Vienna, Austria

Background: Pristane induced lupus (PIL) is an established murine model of induced systemic lupus erythematosus (SLE). Mice develop specific autoantibodies and show symptoms of SLE including arthritis, glomerulonephritis and haemorrhagic pulmonary capillaritis.

Objectives: To investigate the therapeutic effects of in vitro-induced regulatory $T$ cells (iTreg) in the murine model of PIL.

Methods: BALB/c mice were injected i.p. with $0.5 \mathrm{ml}$ of pristane (PIL) or PBS (control). Naive $\mathrm{CD}_{4}^{+}$thymocytes were sorted and cultured and cell suspensions with $>80 \% \mathrm{CD}^{+} \mathrm{FoxP}^{+}$cells (iTreg) were injected i.v. (i) once when PIL was induced $\left(5 \times 10^{6}\right.$ iTreg (iTreg-single) $)$ or (ii) every 4 weeks $\left(1 \times 10^{6}\right.$ iTreg, iTregrep). Mice were monitored for paw swelling and grip strength. After 8 months, histological analysis quantified cartilage degradation, number of osteoclasts, extent of inflammation and bone erosion. Glomerulonephritis and pneumonitis were quantified using the kidney biopsy score and a histomorphometric image analysis system; inflammatory tissue was analysed by tissue cytometry. Serum levels of auto-antibodies were measured by ELISA. Frequencies of B cells, activated and regulatory $\mathrm{CD}_{4}{ }^{+} \mathrm{T}$ cells and Th1, Th2, Th17 cells were measured by flow cytometry. FlowCytomix Pro was used to measure serum cytokines. RT-qPCR was used to measure expression levels of interferon (IFN)-signature and T-cell subset related as well as inflammation-associated genes.

Results: Monthly injections of $1 \times 10^{6}$ iTreg reduced the clinical and histological severity of PIL-arthritis. This was seen by a higher mean grip strength, less mean paw swelling, retardation of symptom onset and a lower summative arthritis severity score (figure A). There was significant reduction of arthritis severity in all histological parameters and in the percentage of affected mice with erosive arthritis (33\% of iTreg-rep mice vs $62 \%$ of PIL-mice). A single boost of $5 \times 10^{6}$ iTreg could not prevent joint manifestation, however a slight retardation in 'loss of grip strength' and significantly less erosive area was seen. In regards to cellular composition of the inflammatory tissue in paws, a significantly increased relative amount of CD4 +Foxp3+cells was seen in the iTreg-rep group compared to PIL group.

Repeatedly injected mice (iTreg-rep) had significantly less renal disease (glomerular activity score) (figure B) and pulmonary involvement (perivascular inflammatory area) (figure C) compared to PIL. iTreg-rep mice had significantly lower serum levels of disease-associated auto-antibodies (figure D). Upon restimulation, splenic $\mathrm{CD}^{+}$cells in iTreg-rep showed a less pronounced Th1, Th2 and Th17 response as well as lower percentages in B- and activated T-cells, whereas the percentage of Treg was higher than in all other groups (figure E). FlowCytomix analysis showed reduced cytokine production after repeated iTreg administration. Correspondingly, iTreg-rep showed decreased expressions of IFN-signature-, Tcell subset- and inflammatory cytokine related genes (figure F).

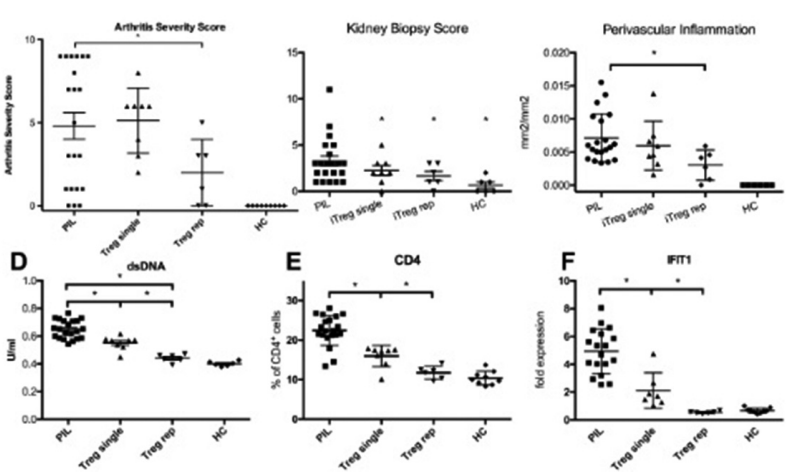

Conclusions: Repeated injections of iTreg ameliorate the clinical, histological, serological as well as the genetic severity of PIL-manifestations. A single boost of iTreg at time of disease induction does not prevent manifestations, but retards the 